\title{
Car Temperature Control System Based on Embedded System
}

\author{
Shuai Sun ${ }^{1, a}$, Xiaoguang $\mathrm{Li}^{2, \mathrm{~b}}$, Huiqin Sun ${ }^{3, \mathrm{c}}$, Tianzi Xue ${ }^{4, \mathrm{~d}}$ and Yongfei Liu ${ }^{5, \mathrm{e}}$ \\ 1,2,3,4,5 Department of Electrical Engineering, Hebei University of Science and Technology, 26 \\ Yuxiang Street, Shijiazhuang, China \\ a18333195575@163.com, bdagenguang@163.com, cshqczx@hebust.edu.cn, \\ dyulingzihbkj@163.com, ${ }^{\mathrm{e}}$ 18730192962@163.com
}

Keywords: air conditioning coach; fuzzy PID; temperature control; $\mu$ C/OS-II; CAN

\begin{abstract}
In view of the temperature control system has nonlinear, time-varying and hysteresis characteristics, and traditional PID control method adaptability poor, robustness is not strong and other issues, using the combination of fuzzy control and PID method, designing temperature control system based on embedded system. Upper machine system using embedded system $\mu \mathrm{C} / \mathrm{OS}-\mathrm{Il}$ to implement unified task creation, scheduling, using CAN bus real-time communication, system anti-jamming is strong, in requiring high precision, fast temperature control occasions with a good prospect.
\end{abstract}

\section{Introduction}

With the rapid progress of society, people's standard of living matter is gradually improving, comfort of human experience results becomes more sought after by people of all ages. Temperature test for the vehicle running status of an important indicator has become a key factor to improve the car comfortable performance. Temperature control this process involves collecting and processing signals. CAN bus is a serial bus, its superior performance and high reliability attracts car designers more and more attention. This article describes the temperature control system based on STM32 and CAN bus design, through the communication of upper machine and lower machine, realizing the temperature data of monitoring, meeting the requirements of high-precision temperature control system.

\section{Air Conditioning Coach Temperature Control System}

\section{Mathematical Model}

According to the law of energy conservation, per unit time into the car interior heat flows minus the unit time car room heat is equal to the car interior heat storage rate, which can get the following equation:

$$
T_{1} \frac{d \Delta \theta_{\alpha}}{d t}+\Delta \theta_{\alpha}=K_{1} \Delta \theta_{f}
$$

$\theta_{\alpha}$-car indoor air temperature; $T_{1}$-adjust the time constant of the object; $K_{1}$-adjust the amplification factor of the object; $\theta_{f}$-interference amount converted to supply air temperature changes.

Considering the actual car room there is a delay, we can get the interference channel transfer function:

$$
\mathrm{W}(\mathrm{s})=\frac{K_{1} e\left(-\tau_{1} s\right)}{T_{1} s+1} .
$$

This equation shows that the temperature control system with nonlinear, time-varying and hysteresis characteristics[1]. 


\section{Establishment Of Control Algorithm}

Because the automobile air conditioning control system time-varying, nonlinear and large disturbance characteristics, PID control algorithm using the premise is the need to set up under the condition of the model has been established, so can not use the traditional PID control algorithm[2]. The fuzzy PID control can be under the premise of accurate model which does not need to know the system, combined with PID control and fuzzy control to adjust PID parameters online. Integrated automotive air conditioning characteristics and to meet the accuracy requirements, we decided to use the fuzzy control and PID combination algorithm[3,4].

Due to the fuzzy control itself is relatively complex, so on the STM32 implemented by using the look-up table method, which can make the corresponding change according to the error and the rate of change of the error, the specific control algorithm flow chart as shown in Fig.1.

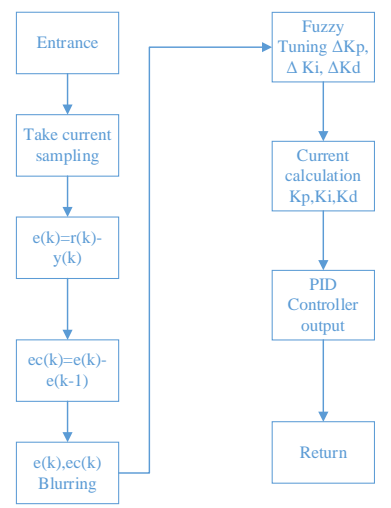

Fig.1 Temperature control algorithm flow chart

\section{The Temperature Control System Hardware Circuit Design}

\section{System Overall Plan Overview}

The system determines to the temperature control system of heating of the air conditioning coach , using upper machine + several lower machines structure, overall system block diagram is shown in Fig.2. Upper machine main achieve the collected temperature processing and issue control instructions to the lower machine via CAN bus, lower machine is placed in the middle of the air conditioning coach, front and rear door. A machine under each major area of multipoint temperature acquisition and to transfer data to upper machine by CAN bus, by receiving the upper machine control command to a PWM wave to control the motor speed to change the ventilation rate to achieve the effect of regulating temperature.

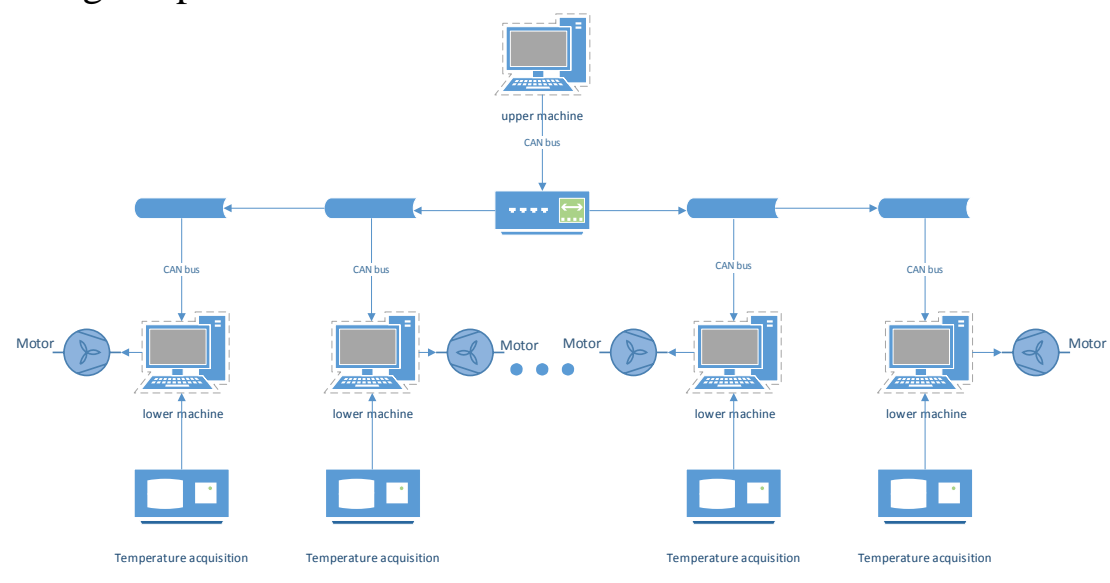

Fig.2 Temperature control system block diagram 
The Upper Computer System

The upper computer controller uses 32 bit processor STM32F103RCT6, which has high-speed data processing ability and memory.

\section{$\mu \mathrm{C} / \mathrm{OS}-\mathrm{II}$ Real-time Operating System}

$\mu \mathrm{C} / \mathrm{OS}-\mathrm{II}$ is ingenious, the structure is simple and refined, which has the characteristics of good portability, cutting, multi-task management, preemptive kernel, task stack, interrupt management, system services, solidification, stability and reliability. For a processor, $\mu \mathrm{C} / \mathrm{OS}$-II transplantation needs to do is modify the part associated with the processor, there are three main files: os_cpu_c.c 、 os_cpu.h、os_cpu_a.asm

(1)os_cpu.h define the data type, the processor related code, declaration function prototype.

(2)os_cpu_c.c mainly completed the task stack and system clock initialization, as well as some of the hook function.

(3)os_cpu_a.asm contains the code needs to be written in assembly.

After the completion of the above work, $\mu \mathrm{C} / \mathrm{OS}$-II system transplant work is completed, then you can create a task on this operating system. Then before start the operating system, we need to define the task priority and trigger mode, after finish the work,we need to define priorities and trigger tasks after the completion of the work, then start the operating system, $\mu \mathrm{C} / \mathrm{OS}-\mathrm{Il}$ is defined based on the priority tasks before scheduling. The following are brief introduction of each user tasks:

(1)AppTaskStart is to start the task, the task of beginning to create the first, after performing hangs himself.

(2)APP_TASK_CAN responsible by CAN bus for data exchange, receive the temperature of the collected data.

(3)APP_TASK_CONTROL task is to control algorithm, after receiving the temperature data, the temperature data is processed by querying the memory of the fuzzy control rule table.

(4)APP_TASK_PWM task is to control the output task, the upper computer output control command, adjust the lower machine output duty cycle of PWM wave to control the effect of the motor.

(5)The APP_TASK_LED is a temperature display task, the upper computer real-time display collected temperature.

Lower Machine System

In order to meet the needs of the CAN bus network interface of data sending and receiving process control, as well as control its components of providing information, lower computer choose macro crystal technology, production of STC15F2K60S2 Series MCU, super anti-interference, in system programming / in the application of programmable without programmer / emulator.

The CAN Communication Circuit Design

The lower machine does not have a CAN controller, the need to connect to the external CAN controller. The lower machine CAN communication circuit is shown in Fig.3.

The upper machine dues to the built-in bxCAN controller, without external CAN controller, simply connected to the CAN transceiver can be connected to the CAN bus hardware parts. The upper machine CAN communication circuit is shown in Fig.4[5].

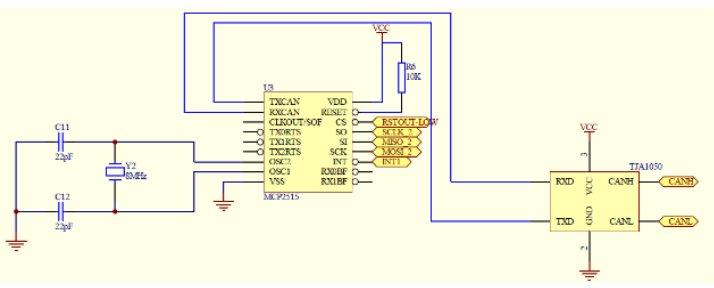

Fig.3 Lower machine CAN communication circuit Fig.4 Upper machine CAN communication circuit 
Brushless DC Motor Drive Circuit

Brushless DC motor needs to be equipped with a drive circuit, to work properly, to complete the corresponding task, this paper brushless motor drive circuit adopts the triode amplifier driver circuit as shown in Fig.5.

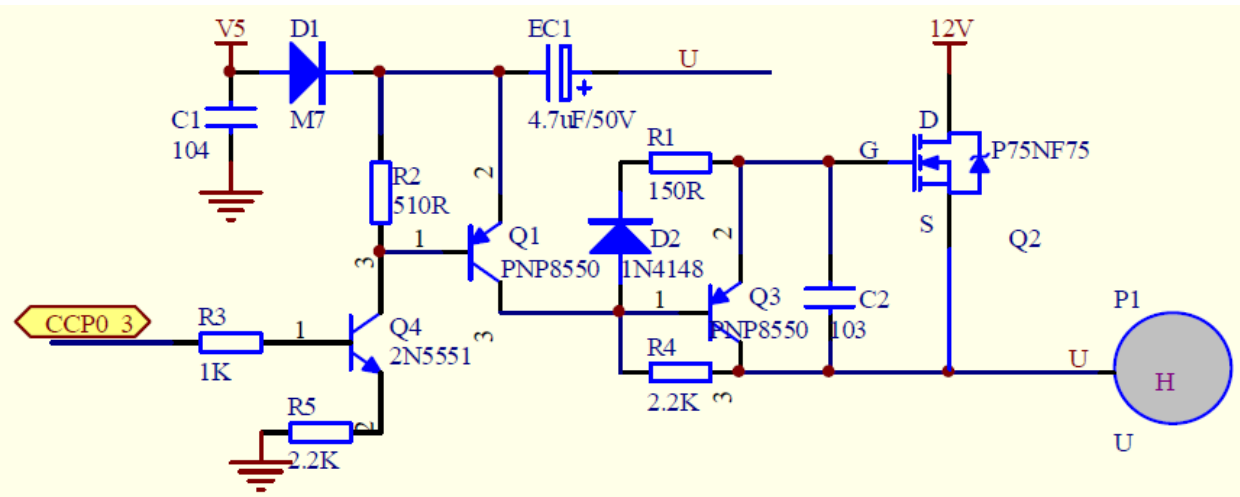

Fig.5 Brushless DC motor drive circuit

Temperature Acquisition Circuit

Lower machine is placed in the middle of the air conditioning coach, front and rear door.A machine under each major area of multipoint temperature acquisition and to transfer data to upper machine by CAN bus, temperature acquisition circuit is shown in Fig.6[6].

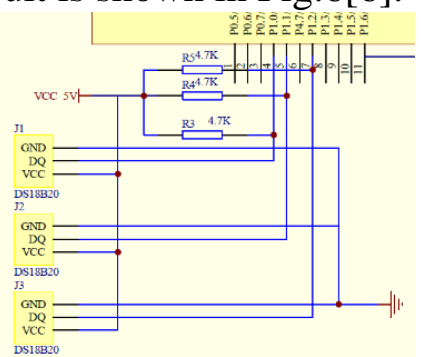

Fig.6 Temperature acquisition circuit

\section{The Temperature Control System Software Program Design}

CAN Communication

The CAN communication is divided into data sending and receiving part, according to the steps of data transmission, the specific flow chart is shown in figure 7 and figure 8.

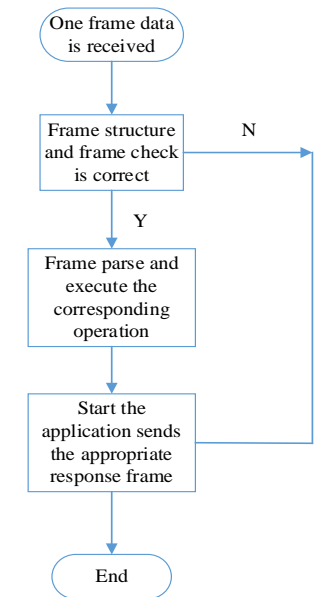

Fig.7 Data reception process

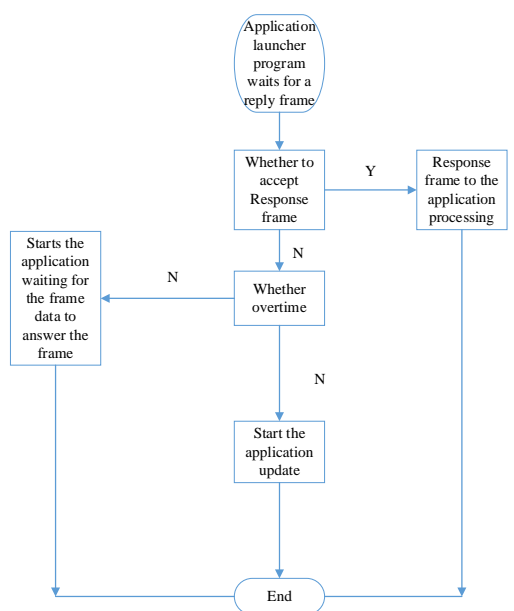

Fig.8 Data transmission process 
Brushless DC Motor Drive

Brushless DC motor speed changes by adjusting the output of the microcontroller PWM wave duty ratio, the concrete software design flow chart is shown in figure 9.

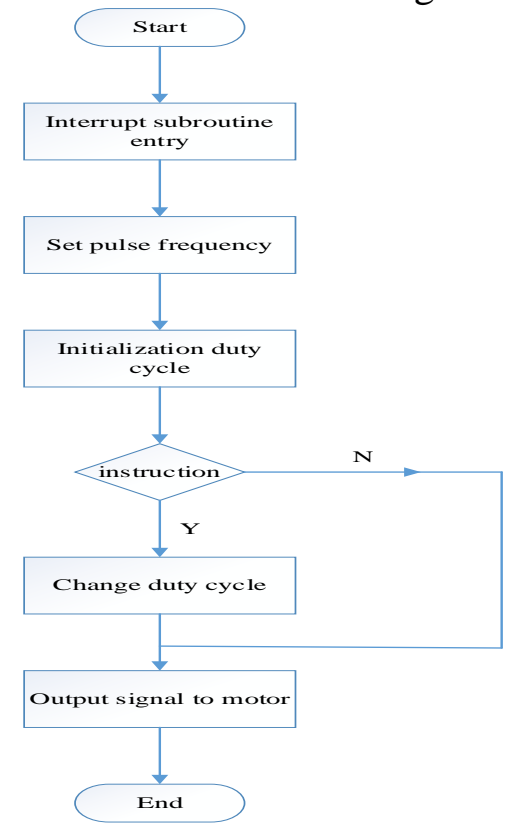

Fig.9 Procedure flow charts of driving circuit

\section{Conclusions}

Based on the research of embedded system, this article designs the real-time temperature control system. Designing a set of high performance, stable operation, strong anti-jamming capability, real-time embedded intelligent temperature control system. This article completed the following work:

(1)For overall design scheme of car control system were summarized,respectively to the upper machine and lower machine system hardware circuit and software design process are expounded.

(2)Completed the $\mu \mathrm{C} / \mathrm{OS}$-II operating system in the STM32 transplant work, in the $\mu \mathrm{C} / \mathrm{OS}$-II real-time operating system on the completion of each user task program design and scheduling work. The design of temperature control system has the ideal system features, in the requirements of high precision, fast control temperature of the occasion has a good application prospect, has a strong practical value.

\section{References}

[1] Letian, Huang: Mic-Computer Information. Forum Vol. 21 (2005),p.70-71.

[2] Lifang Song and Yongli Zhang: Science Technology and Engineering. Forum Vol. 14 (2006),p. 2142-2143.

[3] Leonid Reznik: Engineering Applications of Articial Intelligence. Forum Vol. 13 (2000),p.419-430.

[4] M.Santos and A.L.Dexter: Engineering Pracitice. Forum Vol. 10 (2002),p.1147-1152.

[5] Best.R.J, Morrow.D.J, Laverty.D.M and Crossley.P.A: IEEE Tranactions on Power Delivery. Forum Vol. 25 (2010),p.2835-2841.

[6] Baoping Wang, Mengjun Tian and Xin Wang: Mechatronics. Forum Vol. 6 (2007),p.63-66. 